\title{
Technical note: Is fecal consistency scoring an accurate measure of fecal dry matter in dairy calves?
}

\author{
D. L. Renaud, ${ }^{1 *}$ (ㄴ) L. Buss, ${ }^{2} \odot$ J. N. Wilms, ${ }^{2,3} \oplus$ and M. A. Steele ${ }^{2} \odot$ \\ ${ }^{1}$ Department of Population Medicine, University of Guelph, Guelph, ON, Canada, N1G 2W1 \\ ${ }^{2}$ Department of Animal Biosciences, University of Guelph, Guelph, Ontario, Canada N1G 2W1 \\ ${ }^{3}$ Trouw Nutrition R\&D, 3800 AG, Amersfoort, the Netherlands
}

\section{ABSTRACT}

The objective of this cross-sectional study was to evaluate the accuracy of fecal consistency scoring as a measure of fecal dry matter (DM) in dairy calves. This study was conducted at a commercial grain-fed veal facility in southwestern Ontario. A total of 160 calves arrived at the facility in 2 groups of 80 calves each. Calves were fed milk replacer twice daily at 0700 and $1700 \mathrm{~h}$ and had ad libitum access from arrival onward to water through nipple drinkers and starter through a shared trough. Fecal consistency scores were evaluated once daily in the first $28 \mathrm{~d}$ after arrival before milk feeding. The fecal consistency scoring was conducted using a 4-level scoring scale: $0=$ normal (firm but not hard); 1 = soft (does not hold form, piles but spreads slightly); $2=$ runny (spreads readily); and $3=$ watery (liquid consistency, splatters). Fecal samples were collected from all calves via rectal palpation on d 1, 7, 14, and 21 at $0900 \mathrm{~h}$ for determination of fecal DM. Mixed repeated measures linear regression models were built to assess the accuracy of fecal consistency scoring in predicting fecal DM. Over 4 selected time points (d 1, $7,14$, and 21$)$ the 160 calves were observed, $382(61.6 \%)$ had a fecal consistency score of $0,121(19.5 \%)$ had a score of $1,85(13.7 \%)$ had a score of 2 , and $32(5.2 \%)$ had a score of 3 . A fecal score of 0 had a fecal DM of $25.1 \pm 8.4 \%$, whereas a fecal score of 1 had a DM of $21.8 \pm 8.2 \%$. With respect to calves that had a fecal score of 2 or 3 , their fecal DM was $16.0 \pm 11.1 \%$ and $10.7 \pm 6.9 \%$, respectively. In evaluating the pairwise comparisons generated in the repeated measures model that controlled for day of sampling, a fecal score of 0 had a $3.2 \%, 8.1 \%$, and $12.0 \%$ higher fecal DM, respectively, when compared with those that had a fecal score of 1,2 , and 3 . In addition, calves with a fecal score of 1 had a $5.0 \%$ and $8.8 \%$ higher fecal DM than calves with

Received May 15, 2020.

Accepted July 12, 2020.

*Corresponding author: renaudd@uoguelph.ca a fecal score of 2 and 3, respectively. Finally, calves with a fecal score of 2 had a $3.8 \%$ higher fecal DM than those with a fecal score of 3 . This study confirms that using observational fecal consistency scoring can accurately predict diarrhea or a decline in fecal DM.

Key words: calf, diarrhea, fecal consistency scoring, dry matter

\section{Technical Note}

Diarrhea continues to be a significant issue within the dairy and veal industries as it is the most common reason for morbidity and mortality in calves (Pardon et al., 2012; Urie et al., 2018). The occurrence of diarrhea is also associated with reduced growth, increased age at first calving, and reduced first lactation milk production (Waltner-Toews et al., 1986; Svensson and Hultgren, 2008; Windeyer et al., 2014). Hence, a significant amount of research has focused on identifying methods to prevent this disease from occurring by altering colostrum management (Godden et al., 2019), biosecurity and biocontainment practices (Walker et al., 2012), plane of nutrition (Ollivett et al., 2012; Todd et al., 2017), provision of nutritional supplements (Berge et al., 2009; Signorini et al., 2012; Diraviyam et al., 2014), and environmental cleanliness (McGuirk, 2008; TrotzWilliams et al., 2007).

A commonality in research surrounding diarrhea is the use of fecal consistency scoring to monitor diarrhea prevalence. Outside of research, this method is also commonly used by producers to identify the occurrence of diarrhea (McGuirk, 2008). This scoring was first mentioned by Larson et al. (1977) as a measure to improve uniformity in measuring and reporting in calf experiments. Larson et al. (1977) suggested a score based on fluidity, where a score of 1 is considered normal (firm but not hard, original form is distorted slightly after dropping to floor and settling), 2 is soft (does not hold form, piles but spreads slightly), 3 is runny (spreads readily to about $6 \mathrm{~mm}$ depth), and 4 is watery (liquid consistency, splatters). Kertz and Ches- 
ter-Jones (2004) further cemented this 4-level scoring in a review suggesting that fecal consistency scoring is needed to ensure proper reporting in calf experimental data. McGuirk (2008), however, suggested using a slightly modified 0 to 3 scale, and classified diarrhea as a score of 2 (loose but enough consistency to remain on bedding) or 3 (watery feces that sift through the bedding). Regardless of the scoring system, the majority of studies evaluating diarrhea in dairy calves use a fecal consistency scoring system. To highlight this point, from January to May 2020 (volume 103, issues 1 to 5 of the Journal of Dairy Science), 8 publications that studied calves specifically used some form of fecal consistency scoring.

Despite the relatively common use of fecal consistency scoring, it has not been well validated as a measure of fluidity of feces and can be viewed as a subjective measure. Bellosa et al. (2011) evaluated the accuracy of fecal consistency scoring as a mean to predict percent of DM. On the 22 calves enrolled in the study, they found the higher the fecal consistency score, the lower the percent DM of the feces. However, this study involved a small number of calves that were experimentally inoculated with Cryptosporidium parvum and was not conducted at a commercial setting. Hence, given the relative lack of studies evaluating the ability of fecal consistency scoring to predict the fluidity of feces in a commercial setting, the objective of this cross-sectional diagnostic accuracy study was to evaluate the accuracy of fecal consistency scoring as a measure of fecal DM in dairy calves. Our hypothesis is that fecal consistency scoring would predict the levels of fecal DM.

This study was conducted at a commercial grainfed veal facility in southwestern Ontario. This facility was selected based on proximity to the University of Guelph and willingness to participate in and complete this study. This study is part of a larger experiment that evaluated the provision of an oral antimicrobial on health, growth, and changes in the microbial communities in the gut (L. Buss, T. T. Yohe, L. R. Cangiano, A. J. Keunen, and M. A. Steele, University of Guelph, Guelph, ON, Canada, unpublished data). This study was approved by the University of Guelph Animal Care Committee (Animal Use Protocol \#4134).

Calves arrived at the facility in 2 groups of 80 calves each that arrived on May 13, 2019, and July 15, 2019, respectively. Calves were sourced from local dairy farms and auction facilities. Calves were housed in individual pens $(78 \times 120 \mathrm{~cm})$ on rubber slatted flooring. They were fed a milk replacer $[26 \% \mathrm{CP}, 17 \%$ crude fiber, 4.66 $\mathrm{Mcal} / \mathrm{kg}$ of ME (Mapleview Agri. Ltd., Palmerston, ON, Canada)] twice daily at 0700 and $1500 \mathrm{~h}$. Specifi- cally, calves at each feeding were fed $260 \mathrm{~g}$ of milk replacer in $2 \mathrm{~L}$ of water, $390 \mathrm{~g}$ of milk replacer in $2.5 \mathrm{~L}$ of water, and $455 \mathrm{~g}$ in $3 \mathrm{~L}$ of water from d 0 to 13 , d 14 to 20 , and d 21 to 27 , respectively. In addition, calves had ad libitum access to water through nipple drinkers and starter $(23.67 \%$ CP, $3.72 \%$ crude fiber, $16.58 \% \mathrm{NDF}$, $10.20 \% \mathrm{ADF}, 27.09 \%$ starch, $3.15 \mathrm{Mcal} / \mathrm{kg}$ of $\mathrm{ME}$ ) via a shared trough. A total of $60 \mathrm{~g}$ of a low-sodium electrolyte $[7.5 \% \mathrm{Na}, 0.05 \% \mathrm{Mg}, 1.25 \% \mathrm{~K}$ (Schippers VZ-Powder, Lacombe, AB, Canada)] was mixed into 2 $\mathrm{L}$ and offered to calves following the morning feeding from d 3 to 10 based on the facilities protocol.

Calves were scored for fecal consistency twice daily, immediately before milk feeding, for the first $28 \mathrm{~d}$ at the facility by the same trained research assistant. However, for the purposes of this study only fecal consistency scoring from the morning of $\mathrm{d} 1,7,14$, and 21 was used. The trained observer also scored a group of 80 calves twice on d 10 of a subsequent trial within 40 min of each scoring period to determine intrarater reliability. The scoring system follows Larson et al. (1977) and include 4 levels: $0=$ normal (firm but not hard, original form is distorted slightly after dropping to floor and settling); $1=$ soft (does not hold form, piles but spreads slightly); $2=$ runny (spreads readily); and 3 = watery (liquid consistency, splatters). The fecal consistency scoring system used is visually depicted in Figure 1. The fecal consistency scoring over the entire experimental period is outlined in Figure 2.

Fecal samples were collected from all calves via rectal palpation on d 1, 7, 14, and 21. The first fecal sample was collected the morning after calf arrival at $0900 \mathrm{~h}$, and weekly thereafter after the morning meal between 0900 and $1100 \mathrm{~h}$. Approximately 5 to $15 \mathrm{~g}$ of feces was collected from each calf to be dried for determination of fecal DM. Every 10th sample, a duplicate sample was created where the sample was split into 2 before drying to assess internal reliability. For measurement of fecal DM composition, samples were thawed, placed on tin trays, and dried for $24 \mathrm{~h}$ at $100^{\circ} \mathrm{C}$ (Bellosa et al., 2011). Samples were weighed using a digital weigh scale before and after drying. The percent DM was calculated by dividing the weight of the feces after drying by the weight of the feces before drying.

Statistical analysis was conducted using Stata 14 (StataCorp LLC, College Station, TX). Data were imported from Microsoft Excel (Microsoft Corp., Redmond, WA) into Stata and checked for completion. Descriptive statistics were generated for all variables in the data set. A chi-squared test was used to evaluate differences between categorical variables, whereas a one-way ANOVA with a Tukey post-hoc correction was 


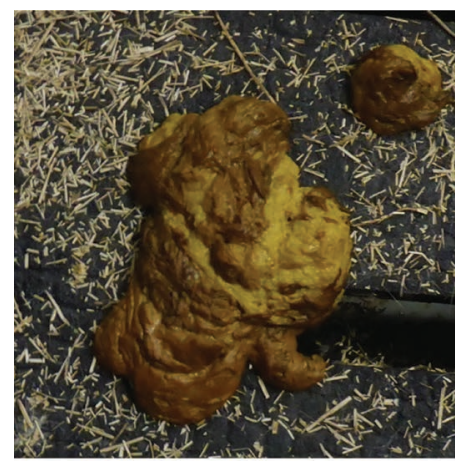

0

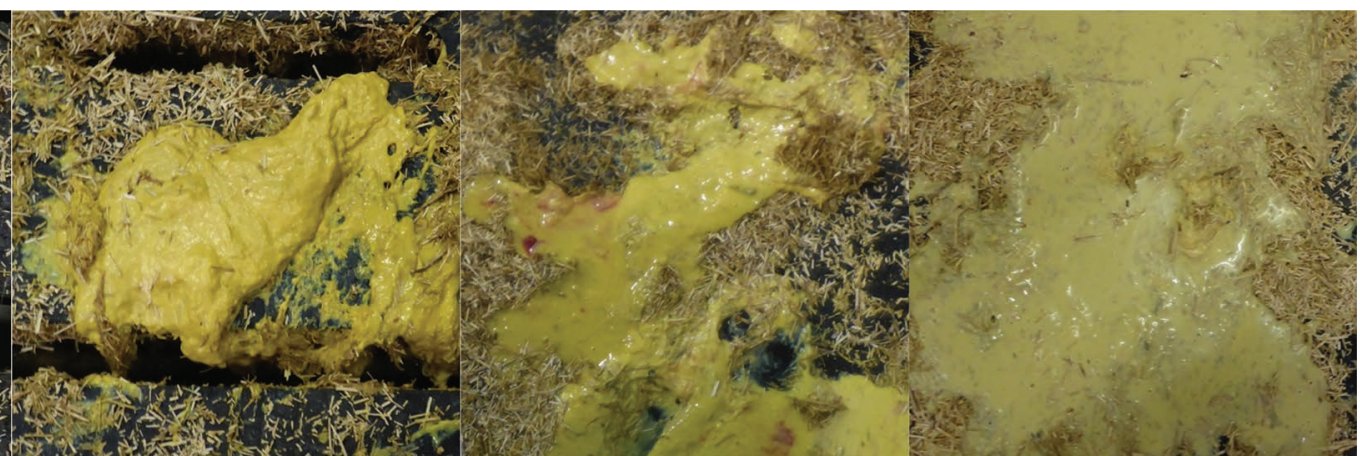

1
2

Figure 1. Visual description of fecal consistency score with each score indicating the following: $0=$ normal (firm but not hard); $1=$ soft $($ does not hold form, piles but spreads slightly); 2 = runny (spreads readily); and $3=$ watery (liquid consistency, splatters). A score of $\geq 2$ indicated the presence of diarrhea.

used to evaluate continuous variables. To determine intrarater reliability, percent agreement (McHugh, 2012) and kappa (Cohen, 1968) were calculated.

A mixed repeated measures linear regression model was built to assess the accuracy of fecal consistency scoring in predicting fecal DM. The dependent variable for the model was fecal DM, and fecal consistency score and day were added as the independent variables. The calf was added in as the repeated measure. The predicted margin was generated for each fecal score to compare the differences between each score. An identical mixed model was also built with fecal DM as the outcome and presence of diarrhea [fecal score of $\geq 2$ (McGuirk, 2008)] and day as independent variables. Homoscedasticity and normality of the residuals and the best linear unbiased predictors were used to evaluate the fit of the models.

A total of 160 calves of an unknown age arrived at the facility. Based on previous work completed in On- tario, it is estimated that the majority of the calves were 3 to $7 \mathrm{~d}$ of age (Renaud et al., 2018). For fecal consistency scoring, the trained research assistant had a $72.2 \%$ agreement and a kappa of 0.54 when scoring a group of 80 calves twice within $40 \mathrm{~min}$ on d 10 of a subsequent trial, suggesting moderate agreement in scoring calves on a 0 to 3 scale. When evaluating the presence of diarrhea ( $\geq 2$ fecal score) or no diarrhea as a dichotomous outcome, the percent agreement improved to $93.7 \%$ and kappa was 0.72 , suggesting substantial agreement. Although not a primary objective of this study, the intrarater reliability is high, with moderate to substantial agreement beyond chance determined. This is an important finding because fecal consistency scoring is sometimes criticized for its subjectivity.

Over the 4 time points the calves were observed for this study, $382(61.6 \%)$ had a fecal consistency score of $0,121(19.5 \%)$ had a score of $1,85(13.7 \%)$ had a score of 2 , and $32(5.2 \%)$ had a score of 3 . Hence,

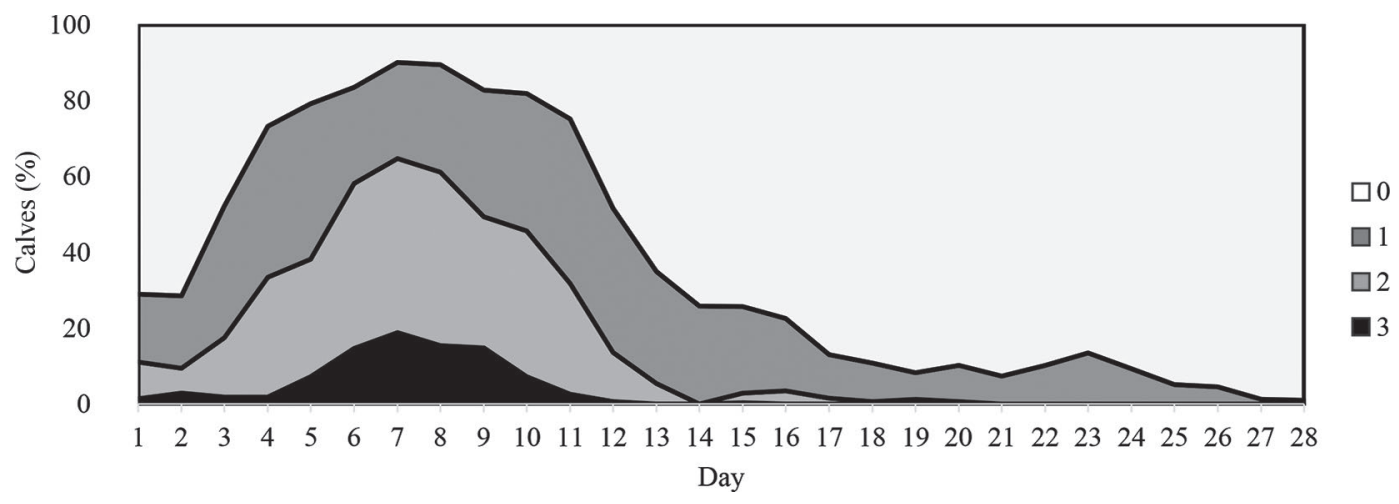

Figure 2. Fecal consistency score from 160 male dairy calves over the first 28 d after arrival to a calf-raising facility. Each fecal consistency score is described as follows: $0=$ normal (firm but not hard); $1=$ soft (does not hold form, piles but spreads slightly); $2=$ runny (spreads readily); and 3 = watery (liquid consistency, splatters). A score of $\geq 2$ indicated the presence of diarrhea. 
$117(18.9 \%)$ of calves were classified as having diarrhea (fecal score of $\geq 2$ ). Fecal consistency score varied over the different observation time points $(P<0.001)$. On d 1, $101(70.1 \%), 25(17.4 \%), 15(10.4 \%)$, and $2(1.4 \%)$ calves had fecal consistency scores of $0,1,2$, and 3 , respectively; however, on $\mathrm{d} 7$, scores were substantially different with 18 (11.2\%), 43 (26.9\%), 69 (43.1\%), and $30(18.8 \%)$ calves having scores of $0,1,2$, and 3 , respectively. At the latter time points, fewer calves had diarrhea. On d 14, 117 (74.0\%), $40(25.3 \%)$, and $1(1.0 \%)$ calf had a fecal score of 0,1 , and 2 , respectively, and on $\mathrm{d} 21,146(92.4 \%)$ and $12(7.6 \%)$ calves had a fecal score of 0 and 1 , respectively.

With respect to fecal DM, the mean over the 4 sampling points was $22.7 \pm 9.7 \%$ (mean $\pm \mathrm{SD}$ ). Similar to fecal score, overall fecal DM differed by sampling time point $(P<0.001)$, with fecal DM being $27.5 \pm 10.7 \%$, $16.3 \pm 10.1 \%, 24.6 \pm 7.5 \%$, and $22.5 \pm 6.6 \%$ on $\mathrm{d} 1,7$, 14 , and 21, respectively. Clear differences were observed in fecal DM between each fecal consistency score as highlighted in Figure 3. Over the 4 time points, a fecal score of 0 had a fecal DM of $25.1 \pm 8.4 \%$, whereas a fecal score of 1 had a DM of $21.8 \pm 8.2 \%$. With respect to calves that had a fecal score of 2 or 3 , their fecal DM was $16.0 \pm 11.1 \%$ and $10.7 \pm 6.9 \%$, respectively. In evaluating the pairwise comparisons generated in the repeated measures model that controlled for day of sampling, a fecal score of 0 had a $3.2 \%$ (95\% CI: 1.5 to $5.0 \% ; P<0.001$ ), $8.1 \%$ (95\% CI: 5.8 to $10.6 \% ; P<$ 0.001 ), and $12.0 \%$ (95\% CI: 8.7 to $15.3 \% ; P<0.001)$ higher fecal DM when compared with those that had a fecal score of 1, 2, and 3, respectively. In addition, calves with a fecal score of 1 had a $5.0 \%$ (95\% CI: 2.6 to $7.3 \% ; P<0.001)$ and $8.8 \%$ (95\% CI: 5.6 to $12.0 \%$; $P<0.001)$ higher fecal DM than calves with a fecal score of 2 and 3 , respectively. Finally, calves with a fecal score of 2 had a $3.8 \%$ (95\% CI: 0.7 to $7.0 \%$; $P=$ 0.02 ) higher fecal DM than those with a fecal score of 3. A separate model comparing fecal DM in calves with diarrhea (defined as a fecal score $\geq 2$ ) to calves without diarrhea (fecal score of $<2$ ) was also built. Calves with diarrhea had a $7.3 \%$ (95\% CI: 5.3 to $9.4 \% ; P<0.001)$ lower fecal DM than calves without diarrhea.

This study highlights that using observational fecal consistency scoring can predict a decline in fecal DM and, thus, that a higher numerical fecal consistency score may be an indicator of increased diarrhea severity. There are some limitations to consider when interpreting the findings of this study. First, this facility fed lower volumes of milk replacer, which could have affected the relationship between fecal score and DM. Hence, additional studies may be necessary to determine the relationship between fecal score and DM when higher volumes of milk are fed. In addition, fecal DM from spot sampling may not represent the overall daily variation of fecal output over 24-h sampling and therefore the overall fecal fluid losses (Fisher and De la Fuente, 1972). Total fecal collection over $24 \mathrm{~h}$ may allow for more precise evaluation of fecal DM but is not applicable in practice. Correlation between fecal $\mathrm{DM}$ and total fecal output per unit of BW remain to be evaluated.

Due to the practical nature of fecal consistency scoring, it is often implemented in primary research studies for assessing diarrhea prevalence. It is also used in research synthesis, with several meta-analyses using fecal consistency scoring as an outcome when evaluating feeding schemes, milk replacer formulations, and supplements fed to calves during the preweaning period (Signorini et al., 2012; Diraviyam et al., 2014; Amado et al., 2019). This scoring system is also commonly used by producers for clinical decision-making, such as when to provide fluid therapy (McGuirk, 2008; Gomez et al., 2017). Hence, this study provides further evidence that fecal consistency scoring has merit as a reliable assessment of fluid loss associated with diarrhea and can be completed with high intrarater reliability in clinical and research conditions.

This work is also in line with Bellosa et al. (2011), who induced diarrhea experimentally using Cryptosporidium parvum oocytes. That study found that fecal consistency scores of 1 to 4 had median fecal DM of $20.9,16.3,9.6$, and $5.8 \%$, respectively. Hence, they had calves with more severe fluid loss compared with the current study, as the corresponding fecal DM were

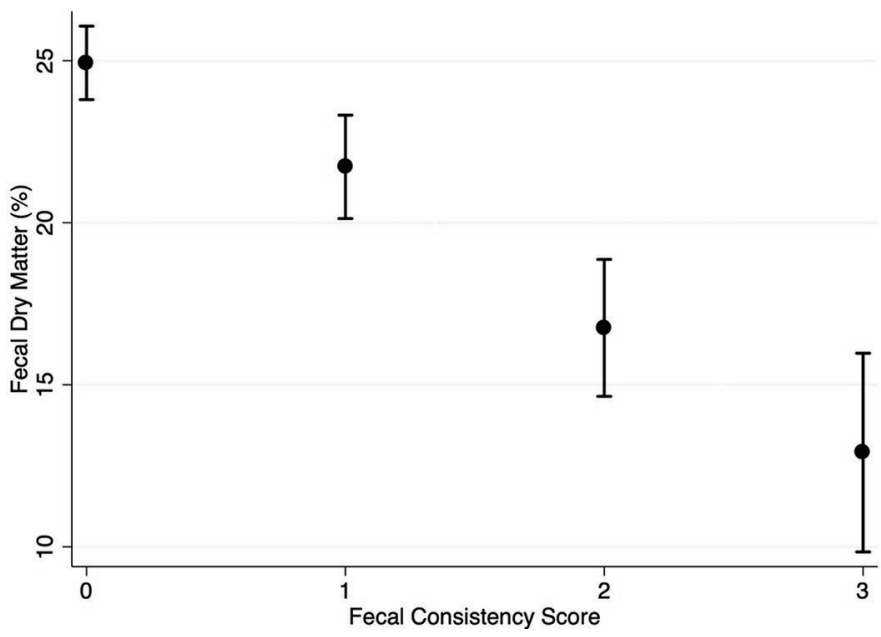

Figure 3. Mean and 95\% CI of fecal DM at each fecal consistency score from 160 calves sampled at d 1, 7, 14, and 21 after arrival to a grain-fed veal facility in southwestern Ontario, Canada. 
higher. This may be associated with the diarrhea etiology differing between both studies. It was shown that diarrhea severity, in terms of fecal water and sodium losses, depends on the age of the patient, the diarrhea etiology, and the stage of the disease (Molla et al., 1981; Raizada et al., 1992). The study conducted by Bellosa et al. (2011), along with the current study, may provide information to determine when diarrheic calves, typically with a fecal score of 3 , require a health intervention. However, other factors, such as percentage of dehydration and milk intakes, may be used to assess whether or not it is needed to initiate oral or intravenous fluid therapy in a diarrheic calf. Fecal scoring may allow for early detection of diarrhea, and thus early intervention, which would mitigate severity of dehydration and metabolic acidosis.

As fecal consistency scoring is commonly used as a metric for diarrhea in most studies evaluating health in dairy calves, it is necessary to validate this technique. This study confirms that using fecal consistency scoring can be used as a metric for evaluating fecal DM. Further studies should be encouraged to continue using this measure; however, because few studies have reported intra- or interrater reliability of this measure, researchers should be encouraged to calculate this to ensure that the scoring used is repeatable.

\section{ACKNOWLEDGMENTS}

The authors thank the Natural Sciences and Engineering Research Council of Canada (Ottawa, ON, Canada), Trouw Nutrition (Guelph, ON, Canada), Bayer Animal Health (Mississauga, ON, Canada), Alberta Milk (Edmonton, AB, Canada), Lallemand (Montreal, QC, Canada), Westgen (Abbotsford, BC, Canada), BC Dairy Association (Burnaby, BC, Canada), SaskMilk (Regina, SK, Canada), and the Dairy Farmers of Manitoba (Winnipeg, MB, Canada) for the financial support provided for this study. The authors have not stated any conflicts of interest.

\section{REFERENCES}

Amado, L., H. Berends, L. N. Leal, J. Wilms, H. Van Laar, W. J. J. Gerrits, and J. Martín-Tereso. 2019. Effect of energy source in calf milk replacer on performance, digestibility, and gut permeability in rearing calves. J. Dairy Sci. 102:3994-4001. https://doi.org/10 .3168/jds.2018-15847.

Bellosa, M. L., D. V. Nydam, J. L. Liotta, J. A. Zambriski, T. C. Linden, and D. D. Bowman. 2011. A comparison of fecal percent dry matter and number of Cryptosporidium parvum oocysts shed to observational fecal consistency scoring in dairy calves. J. Parasitol. 97:349-351. https://doi.org/10.1645/GE-2475.1.

Berge, A. C. B., T. E. Besser, D. A. Moore, and W. M. Sischo. 2009. Evaluation of the effects of oral colostrum supplementation dur- ing the first 14 days on the health and performance of preweaned calves. J. Dairy Sci. 92:286-295. https://doi.org/10.3168/jds.2008 $-1433$.

Cohen, J. 1968. Weighted kappa: Nominal scale agreement with provision for scaled disagreement or partial credit. Psychol. Bull. 70:213-220.

Diraviyam, T., B. Zhao, Y. Wang, R. Schade, A. Michael, and X. Zhang. 2014. Effect of chick egg yolk antibodies (IgY) against diarrhea in domesticated animals: A systematic review and metaanalysis. PLoS One 9:e97716. https://doi.org/10.1371/journal pone.0097716.

Fisher, E. W., and G. H. De la Fuente. 1972. Water and electrolyte studies in newborn calves with particular reference to the effects of diarrhoea. Res. Vet. Sci. 13:315-323. https://doi.org/10.1016/ S0034-5288(18)34007-4.

Godden, S. M., J. E. Lombard, and A. R. Woolums. 2019. Colostrum management for dairy calves. Vet. Clin. North Am. Food Anim. Pract. 35:535-556. https://doi.org/10.1016/j.cvfa.2019.07.005.

Gomez, D. E., L. G. Arroyo, Z. Poljak, L. Viel, and J. S. Weese. 2017. Implementation of an algorithm for selection of antimicrobial therapy for diarrhoeic calves: Impact on antimicrobial treatment rates, health and faecal microbiota. Vet. J. 226:15-25. https://doi .org/10.1016/j.tvjl.2017.06.009.

Kertz, A. F., and H. Chester-Jones. 2004. Invited Review: Guidelines for measuring and reporting calf and heifer experimental data. J. Dairy Sci. 87:3577-3580. https://doi.org/10.3168/jds.S0022 -0302(04)73495-5.

Larson, L. L., F. G. Owen, R. D. Albright, R. D. Appleman, R. C. Lamb, and L. D. Muller. 1977. Guidelines toward more uniformity in measuring and reporting calf experimental data. J. Dairy Sci. 60:989-991. https://doi.org/10.3168/jds.S0022-0302(77)83975-1.

McGuirk, S. M. 2008. Disease management of dairy calves and heifers. Vet. Clin. North Am. Food Anim. Pract. 24:139-153. https://doi .org/10.1016/j.cvfa.2007.10.003.

McHugh, M. L. 2012. Interrater reliability: The kappa statistic. Biochem. Med. (Zagreb) 22:276-283

Molla, A. M., M. Rhman, S. A. Sarker, D. A. Sack, and A. Molla. 1981. Stool electrolyte content and purging rates in diarrhea caused by rotavirus, enterotoxigenic $E$. coli and $V$. cholerae in children. J. Pediatr. 98:835-838. https://doi.org/10.1016/S0022 $-3476(81) 80863-3$.

Ollivett, T. L., D. V. Nydam, T. C. Linden, D. D. Bowman, and M. E. Van Amburgh. 2012. Effect of nutritional plane on health and performance in dairy calves after experimental infection with Cryptosporidium parvum. J. Am. Vet. Med. Assoc. 241:1514-1520. https: //doi.org/10.2460/javma.241.11.1514.

Pardon, B., K. De Bleecker, M. Hostens, J. Callens, J. Dewulf, and P. Deprez. 2012. Longitudinal study on morbidity and mortality in white veal calves in Belgium. BMC Vet. Res. 8:26. https://doi.org/ 10.1186/1746-6148-8-26.

Raizada, N., R. C. Bhatia, B. K. Jain, and H. Singh. 1992. Stool electrolytes in acute dehydrating gastroenteritis. Indian Pediatr. 29:461-465.

Signorini, M. L., L. P. Soto, M. V. Zbrun, G. J. Sequeira, M. R. Rosmini, and L. S. Frizzo. 2012. Impact of probiotic administration on the health and fecal microbiota of young calves: A meta-analysis of randomized controlled trials of lactic acid bacteria. Res. Vet. Sci. 93:250-258. https://doi.org/10.1016/j.rvsc.2011.05.001.

Svensson, C., and J. Hultgren. 2008. Associations between housing, management, and morbidity during rearing and subsequent first lactation milk production of dairy cows in southwest Sweden. J. Dairy Sci. 91:1510-1518. https://doi.org/10.3168/jds.2007-0235.

Todd, C. G., K. E. Leslie, S. T. Millman, V. Bielmann, N. G. Anderson, J. M. Sargeant, and T. J. DeVries. 2017. Clinical trial on the effects of a free-access acidified milk replacer feeding program on the health and growth of dairy replacement heifers and veal calves. J. Dairy Sci. 100:713-725. https://doi.org/10.3168/jds.2016-11401.

Trotz-Williams, L. A., S. W. Martin, K. E. Leslie, T. Duffield, D. V. Nydam, and A. S. Peregrine. 2007. Calf-level risk factors for 
neonatal diarrhea and shedding of Cryptosporidium parvum in Ontario dairy calves. Prev. Vet. Med. 82:12-28. https://doi.org/10 .1016/j.prevetmed.2007.05.003.

Urie, N. J., J. E. Lombard, C. B. Shivley, C. A. Kopral, A. E. Adams, T. J. Earleywine, J. D. Olson, and F. B. Garry. 2018. Preweaned heifer management on US dairy operations: Part V. Factors associated with morbidity and mortality in preweaned dairy heifer calves. J. Dairy Sci. 101:9229-9244. https://doi.org/10.3168/jds .2017-14019.

Walker, W. L., W. B. Epperson, T. E. Wittum, L. K. Lord, P. J. Rajala-Schultz, and J. Lakritz. 2012. Characteristics of dairy calf ranches: Morbidity, mortality, antibiotic use practices, and biosecurity and biocontainment practices. J. Dairy Sci. 95:2204-2214. https://doi.org/10.3168/jds.2011-4727.

Waltner-Toews, D., S. W. Martin, A. H. Meek, and I. McMillan. 1986. Dairy calf management, morbidity, and mortality in Ontario Hol- stein herds. I. The data. Prev. Vet. Med. 4:103-124. https://doi .org/10.1016/0167-5877(86)90017-6.

Windeyer, M. C., K. E. Leslie, S. M. Godden, D. C. Hodgins, K. D. Lissemore, and S. J. LeBlanc. 2014. Factors associated with morbidity, mortality, and growth of dairy heifer calves up to 3 months of age. Prev. Vet. Med. 113:231-240. https://doi.org/10.1016/j .prevetmed.2013.10.019.

\section{ORCIDS}

D. L. Renaud (1) https://orcid.org/0000-0002-3439-3987

L. Buss (ㄴ) https://orcid.org/0000-0002-5648-2745

J. N. Wilms ๑ https://orcid.org/0000-0003-1725-1087

M. A. Steele ๑ https://orcid.org/0000-0001-6941-6205 